\title{
Orientation Dependent Interlayer Stacking Structure in
}

\section{Bilayer $\mathrm{MoS}_{2}$ domains}

Shanshan Wang ${ }^{1}$, Hidetaka Sawada ${ }^{1,2,3}$, Christopher S. Allen ${ }^{1,3}$, Angus I. Kirkland ${ }^{1,3}$, Jamie H.

$$
\text { Warner }^{1 *}
$$

${ }^{1}$ Department of Materials, University of Oxford, Parks Road, Oxford, OX1 3PH, United Kingdom

${ }^{2}$ JEOL Ltd., 3-1-2 Musashino, Akishima, Tokyo 196-8558, Japan

${ }^{3}$ Electron Physical Sciences Imaging Center, Diamond Light Source Ltd, Didcot, Oxfordshire, OX11 0DE, United Kingdom

*Jamie.warner@materials.ox.ac.uk;

\begin{abstract}
We have studied the atomic structure of small secondary domains that nucleate on monolayer $\mathrm{MoS}_{2}$ grown by chemical vapour deposition (CVD), which form the basis of bilayer $\mathrm{MoS}_{2}$. The small secondary bilayer domains have a faceted geometry with three-fold symmetry and adopt two distinct orientations with $60^{\circ}$ rotation relative to an underlying monolayer $\mathrm{MoS}_{2}$ single crystal sheet. The two distinct orientations are associated with the $2 \mathrm{H}$ and $3 \mathrm{R}$ stacking configuration for bilayer $\mathrm{MoS}_{2}$. Atomic resolution images have been recorded using high angle annular dark field scanning transmission electron microscopy (HAADF-STEM) that show the edge termination, lattice orientation and stacking sequence of the bilayer domains relative to the underlying monolayer $\mathrm{MoS}_{2}$. These results provide important insights that bilayer $\mathrm{MoS}_{2}$ growth from $60^{\circ}$ rotated small nuclei on the surface of monolayer $\mathrm{MoS}_{2}$ could lead to defective boundaries when merged to forming larger continuous bilayer regions and that pure $\mathrm{AA}^{\prime}$ or $\mathrm{AB}$ bilayer stacking may be challenging unless from a single seed.
\end{abstract}


KEYWORDS: $\mathrm{MoS}_{2}$, HAADF-STEM, 2D materials, bilayers

Layered molybdenum disulphide $\left(\mathrm{MoS}_{2}\right)$ has recently attracted interest due to its direct bandgap in single layer form, which exhibits semiconducting performance and expands the potential application of $2 \mathrm{D}$ materials in nanoelectronics and optoelectronics. ${ }^{1-4}$ Monolayer $\mathrm{MoS}_{2}$ has Mo atoms bonded to $\mathrm{S}$ atoms in a trigonal prismatic coordination by strong covalent bonds, while the interactions between layers are due to weak van der Waals (vdW) forces. This layered configuration gives rise to polytypism in few-layer $\mathrm{MoS}_{2}$ with different stacking structures. A change in $\mathrm{MoS}_{2}$ from monolayer to bilayer modifies the band structure, providing tunability in electrical, optical, vibrational and chemical properties. $^{5-8}$ It has been reported that few-layer $\mathrm{MoS}_{2}$ shows higher carrier mobility, ${ }^{9,10}$ better chemical stability ${ }^{11,12}$ and a more sensitive field-induced effect ${ }^{13,14}$ in transistors than its monolayer counterpart. Moreover, the bandgap of bilayer $\mathrm{MoS}_{2}$ can be tuned by applying a vertical electric field. ${ }^{15}$ For these reasons, few-layer $\mathrm{MoS}_{2}$ is a good candidate for valley-tronics due to the degree of freedom offered by additional layers. ${ }^{16,17}$

The stacking order between adjacent $\mathrm{MoS}_{2}$ layers has been shown to be one of the major factors that influence the properties of few-layer $\mathrm{MoS}_{2}$ properties in several experimental and theoretical studies. Different stacking sequences modify the crystal symmetry, which varies the Coulomb interaction between layers resulting in different equilibrium interlayer distances. ${ }^{5,18}$ Therefore, many physical properties of 2D layered materials including bandgap size, phonon vibration frequency, magnetism and superconductivity can be manipulated by altering the layer stacking configuration. ${ }^{19,20}$ The most energetically favourable polytypes of few-layer $\mathrm{MoS}_{2}$ are $2 \mathrm{H}$ (space group: $P 6_{3} / m m c$ ) and 3R (space group: $R 3 m$ ) (Figure 1a), which have the same intralayer coordination (trigonal prismatic coordination between the Mo and adjacent $\mathrm{S}$ atoms) but with different interlayer stacking. ${ }^{21}$

Fabricating few-layer $\mathrm{MoS}_{2}$ with specific stacking order can be achieved by two main routes: (i) sequential mechanically stacking of $\mathrm{MoS}_{2}$ monolayers with a precise control over twist angles; ${ }^{22}$ 
(ii) growth of few-layer $\mathrm{MoS}_{2}$ followed by a selection of quantified stacking sequences. ${ }^{18,19,23}$ Direct growth of few-layer $\mathrm{MoS}_{2}$ crystals has the advantage of scalable production and no interlayer contamination and in particular, chemical vapour deposition (CVD) growth of $\mathrm{MoS}_{2}$ has been achieved with wafer scale coverage. ${ }^{24-27} \mathrm{CVD}$ growth of monolayer $\mathrm{MoS}_{2}$ is often carried out using amorphous substrates such as $\mathrm{SiO}_{2} / \mathrm{Si}$ wafers, whereas the second layer of $\mathrm{MoS}_{2}$ grows on the crystalline monolayer $\mathrm{MoS}_{2}$ surface. Compared to the intensive study of monolayer $\mathrm{MoS}_{2}$, research on the growth of few-layer $\mathrm{MoS}_{2}$ and corresponding structural analysis is limited.

In addition to stacking sequences, the edge is another significant structural parameter that can influence the electrical, optical, magnetic and catalytic properties of 2D materials. Fabricating specific edge structures in graphene nanoribbons can open up a band gap and create unique spin states under the influence of an electric field. ${ }^{28,29}$ Moreover, study on edges facilitates an in-depth understanding of growth mechanisms at the atomic scale and can help correlate the synthesis (precursor concentration, substrate, etc.) with the resultant $2 \mathrm{D}$ material structure. ${ }^{30,31}$ There have also been several theoretical reports relating the edge structure of $\mathrm{MoS}_{2}$ to its properties, ${ }^{32-34}$ including the prediction of a ferromagnetic ground state for Mo-terminated edges as well as metallic and semiconducting behaviour in zigzag and armchair $\mathrm{MoS}_{2}$ nanoribbons. ${ }^{35}$ Experimentally, aberration-corrected transmission electron microscopy (AC-TEM) in phase contrast imaging mode and scanning transmission electron microscopy (AC-STEM) can determine edge structures. ${ }^{36-39}$ High angle annular dark field STEM (HAADF-STEM) is particularly suitable for structural studies of 2D multi-element materials, such as $\mathrm{MoS}_{2}$, due to its z-dependent contrast. ${ }^{40,41}$ Mo- and S-zigzag edges are most commonly observed in as-grown monolayer $\mathrm{MoS}_{2}$, with several reconstructed configurations having been observed with different S coverage or displacements in the outermost atomic rows. ${ }^{39}$ The structure and evolution of torn edges as the defect concentration increases in cracked monolayer $\mathrm{MoS}_{2}$ samples have also been investigated. $^{42}$ 
Here we use aberration corrected HAADF-STEM to study the atomic structure of smaller secondary domains of $\mathrm{MoS}_{2}$ that nucleate on top of a larger underlying monolayer single crystal grown by CVD. We examine the crystal shape, stacking order and the step edge configurations of the $\mathrm{MoS}_{2}$ secondary domains relative to the underlying larger $\mathrm{MoS}_{2}$ monolayer. The $\mathrm{MoS}_{2}$ secondary layers mostly adopt a three-pointed geometry, similar to a shuriken and are epitaxially matched to the $\mathrm{MoS}_{2}$ monolayers in both $2 \mathrm{H}$ and $3 \mathrm{R}$ phases. The atomic configurations on step edges for differently stacked second layers are various, with 50\% S-covered Mo Klein edges for $2 \mathrm{H}$ stacking and Mo-terminated zigzag edges for 3R stacking. The growth mechanism for these epitaxially stacked shuriken shaped $\mathrm{MoS}_{2}$ second layers are also investigated, indicating the kinetic controlled growth conditions and a substrate influence from the bottom crystalline $\mathrm{MoS}_{2}$ monolayer.

\section{Results and discussion}

For bilayer $\mathrm{MoS}_{2}$, there are five possible high-symmetry stacking sequences, denoted as AA, AA', $\mathrm{AB}, \mathrm{AB}$ ' and $\mathrm{A}^{\prime} \mathrm{B}$, consistent with the nomenclature for bilayer hexagonal boron nitride $(h$ $\mathrm{BN}){ }^{5,43}$ The $2 \mathrm{H}$ and $3 \mathrm{R}$ polytypes of $\mathrm{MoS}_{2}$ bilayers correspond to $\mathrm{AA}$ ' and $\mathrm{AB}$ stacking (Figure 1a), which are the most energetically stable and commonly observed phases in natural and synthetic materials. For AA' stacking, the Mo (S) atoms in one layer overlap with the S (Mo) atoms in the other layer. The lattice orientation between the two layers is rotated by $60^{\circ}$ with all atoms in the two layers fully eclipsed. AB stacking consists of two staggered layers with $0^{\circ}$ rotation, where double stacked $\mathrm{S}$ atoms in one layer are located above (below) the centers of the hexagonal rings in the other layer. In this configuration three different atomic columns are observed in the top down projection; a single Mo atom, two S atoms plus one Mo atom, and two $\mathrm{S}$ atoms, respectively, marked as 1', 2' and 3' in Figure 1a. These two stacking sequences can be interconverted through rotation around an axis, perpendicular to the sheet as shown in Figure 1c. 
Figure $1 \mathrm{~b}$ shows an HAADF-STEM image of several smaller $\mathrm{MoS}_{2}$ domains on the surface of a larger single crystalline monolayer. The small $2^{\text {nd }}$ layer nuclei have a triangular geometry, as indicated by green dashed circles, while the larger ones have a three-pointed shuriken-like shape. The larger secondary domains align along two different orientations with a $60^{\circ}$ rotation, which are identified by three tips of each domain and labelled by yellow and red markers, respectively. Mo zigzag and S zigzag structures have been reported as the two most energetically favourable edge terminations in monolayer $\mathrm{MoS}_{2},{ }^{44}$ and we therefore propose two alternative structures that could lead to the generation of these two types of larger $2^{\text {nd }}$-layer domains as shown in Figure 1d. We suggest that either the domains arise from different sub-lattice sites with the same $2^{\text {nd }}$-layer lattice orientation, in which case one triangle is S zig-zag edge terminated and the other is Mo zig-zag edge terminated (left panel of Figure 1d) (leading to $\mathrm{AA}^{\prime}$ and $\mathrm{AB}^{\prime}$ ' stacking), or that they adopt different $2^{\text {nd }}$-layer lattice orientations, with a $60^{\circ}$ rotation, in which case both have the same Mo zig-zag sub-lattice edge terminations (right panel of Figure 1d) (leading to AA' and AB stacking). 

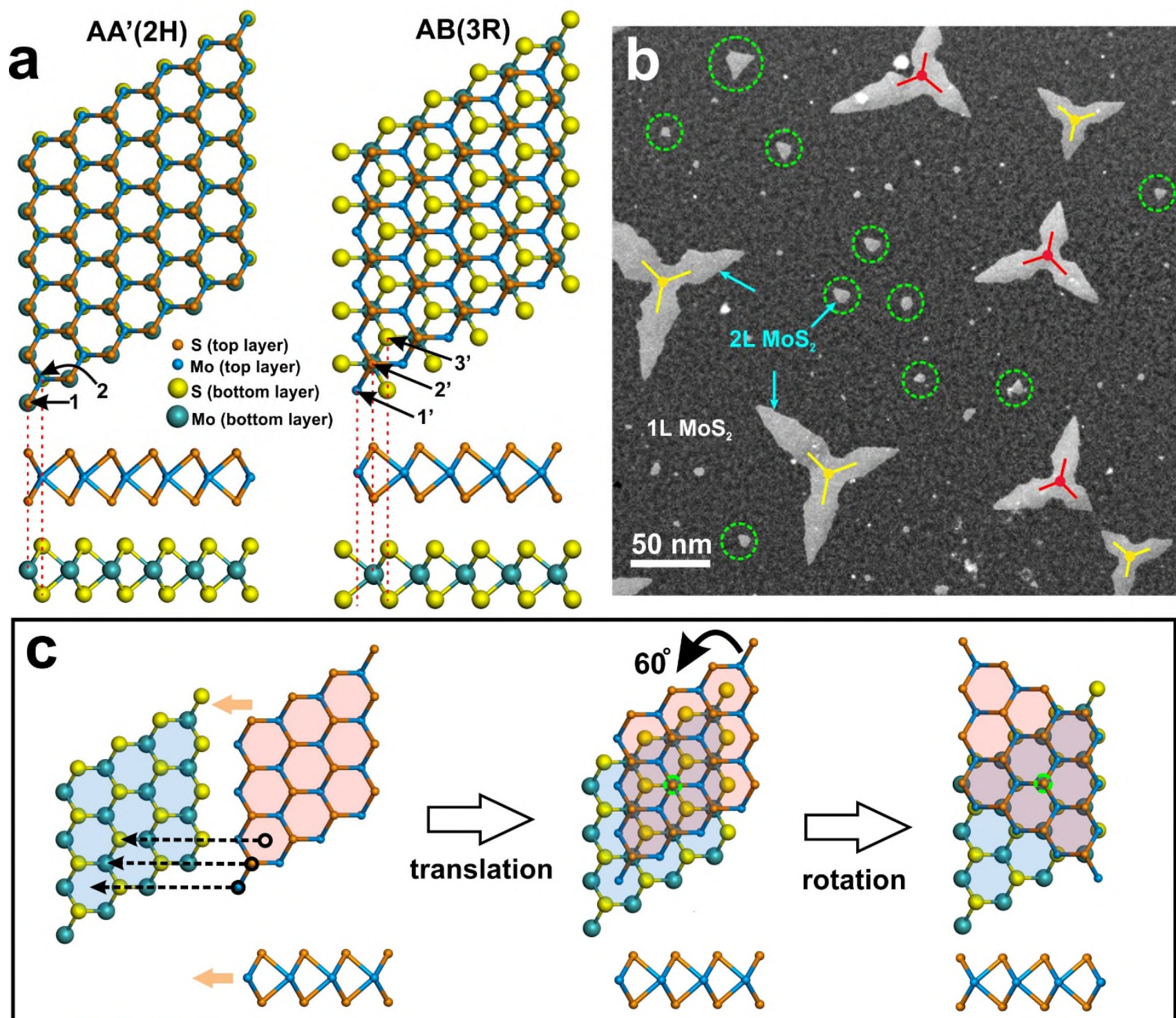

$$
8888 \%
$$
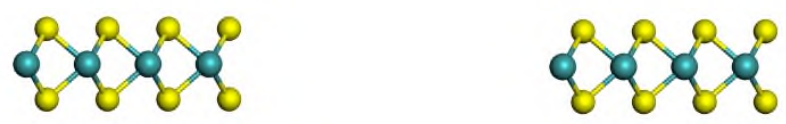

AB stack

AA' stack

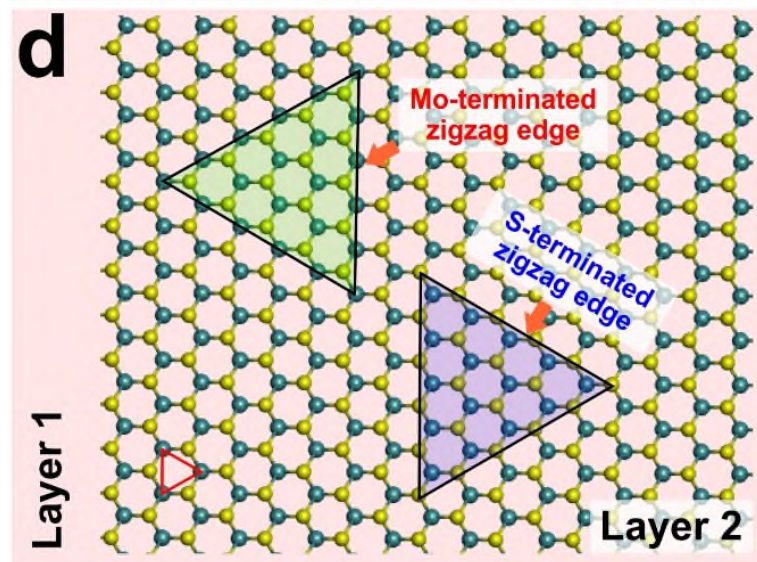

og og og og og bo og bo bo bo

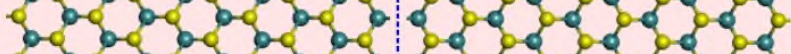

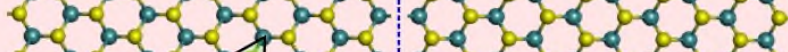

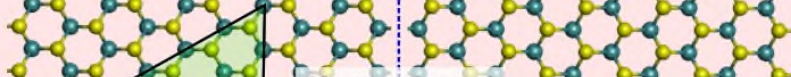

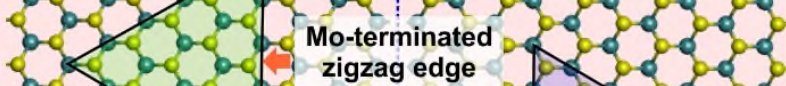

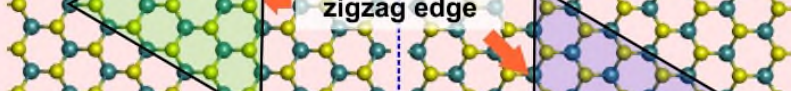

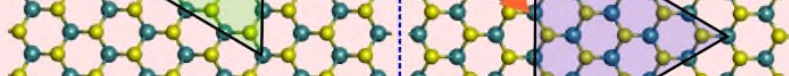

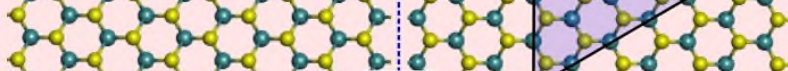
bo oo o $60^{\circ}$ o 00 o bo oo of o o of o o o o Layer 2 od oq o o o o Layer 2 
Figure 1. (a) Schematic illustration of $2 \mathrm{H}$ and $3 \mathrm{R}$ polytypes in $\mathrm{MoS}_{2}$ bilayers, corresponding to $A A^{\prime}$ and $A B$ stacking sequences. Orange, blue, yellow and green spheres in the atomic model indicate $\mathrm{S}$ atoms in the top $\mathrm{MoS}_{2}$ layer, Mo atoms in the top $\mathrm{MoS}_{2}$ layer, $\mathrm{S}$ atoms in the bottom $\mathrm{MoS}_{2}$ layer and Mo atoms in the bottom $\mathrm{MoS}_{2}$ layer, respectively. (b) Low magnification HAADF-STEM image showing three-pointed shuriken-shaped $\mathrm{MoS}_{2}$ bilayers on single-crystalline monolayer $\mathrm{MoS}_{2}$ in two different epitaxial orientations with a $60^{\circ}$ rotation. Domains with two different stacking sequences are labelled by yellow and red marks, respectively. There are also distributions of small nuclei in the vicinity of the larger domains, indicated by green dashed circles. (c) Schematic diagram showing the transformation between $\mathrm{AB}$ and $\mathrm{AA}^{\prime}$ stacking. The top and bottom $\mathrm{MoS}_{2}$ monolayer are indicated by a red and a blue background, respectively, so that the stacked bilayer region can be visualised as a color difference. The center of the $60^{\circ}$ rotation from the $\mathrm{AB}$ to the $\mathrm{AA}$ ' sequence is marked by a green dashed circle. (d) Schematic illustration showing two possible structural relationships between two larger $2^{\text {nd }}$-layer $\mathrm{MoS}_{2}$ domains rotated by $60^{\circ}$. The red background represents the $1^{\text {st }}-$ layer of the underlying single-crystalline $\mathrm{MoS}_{2}$ film.

Figure 2a shows the tip region of a $2^{\text {nd }}$-layer $\mathrm{MoS}_{2}$ crystal (highlighted by a yellow box inset) corresponding to the shuriken-shaped domain outlined by a dashed red triangle in Figure 1b. An obvious contrast difference can be seen between the bilayer and the monolayer region. The slight contrast non-uniformity across the HAADF-STEM image originates from residual hydrocarbon contamination absorbed on the specimen. The tip of the domain is not atomically sharp and also shows uneven edges. Figure $2 \mathrm{~b}$ shows an extended step edge of the $2^{\text {nd }}$-layer $\mathrm{MoS}_{2}$, corresponding to the region indicated by the blue arrow in the inset to Figure 2a together with the power spectrum calculated from Figure 2b with white circles indicating $\{100\}$ reflections (zigzag lattice orientation) and green circles corresponding to $\{110\}$ reflections (armchair lattice orientation). The HAADF image and its corresponding power spectrum indicate that the long $2^{\text {nd }}$-layer step edge predominantly aligns along the zigzag lattice direction with occasional deflections and small terraces. Figure $2 \mathrm{c}$ shows a magnified image taken from the region marked with a white box in Figure 2a with the corresponding atomic model and multislice HAADF image simulation shown in Figures $2 \mathrm{~d}$ and 2e, respectively. Intensity 
line profiles across two adjacent lattice rows through the $2^{\text {nd }}$-layer $\mathrm{MoS}_{2}$ step edge, labelled by 1 and 2 in Figure 2c and 2e, are measured for both experimental and simulated HAADF images, showing a good agreement (Figure $2 \mathrm{f}$ and $2 \mathrm{~g}$ ). The HAADF-STEM image indicates that this bilayer region exhibits AA' stacking. Most step edges of AA' stacked 2 ${ }^{\text {nd }}$-layer $\mathrm{MoS}_{2}$ adopt the $50 \%$ S-covered Mo Klein edge structure with single dangling S atoms at Mo-terminated zigzag edges, as shown in Figure $2 \mathrm{~d}$. The attachment of these single $\mathrm{S}$ atoms to edges is also supported by the intensity line profiles in Figure 2f, highlighted by the blue arrow (Figure S1). We also observed a small background intensity fluctuation in the monolayer region close to the $2^{\text {nd }}$-layer step edge in the experimental HAADF-STEM image, as shown by the black dashed lines in Figure $2 \mathrm{f}$ which could be attributed to residual $\mathrm{C}$ contamination. 

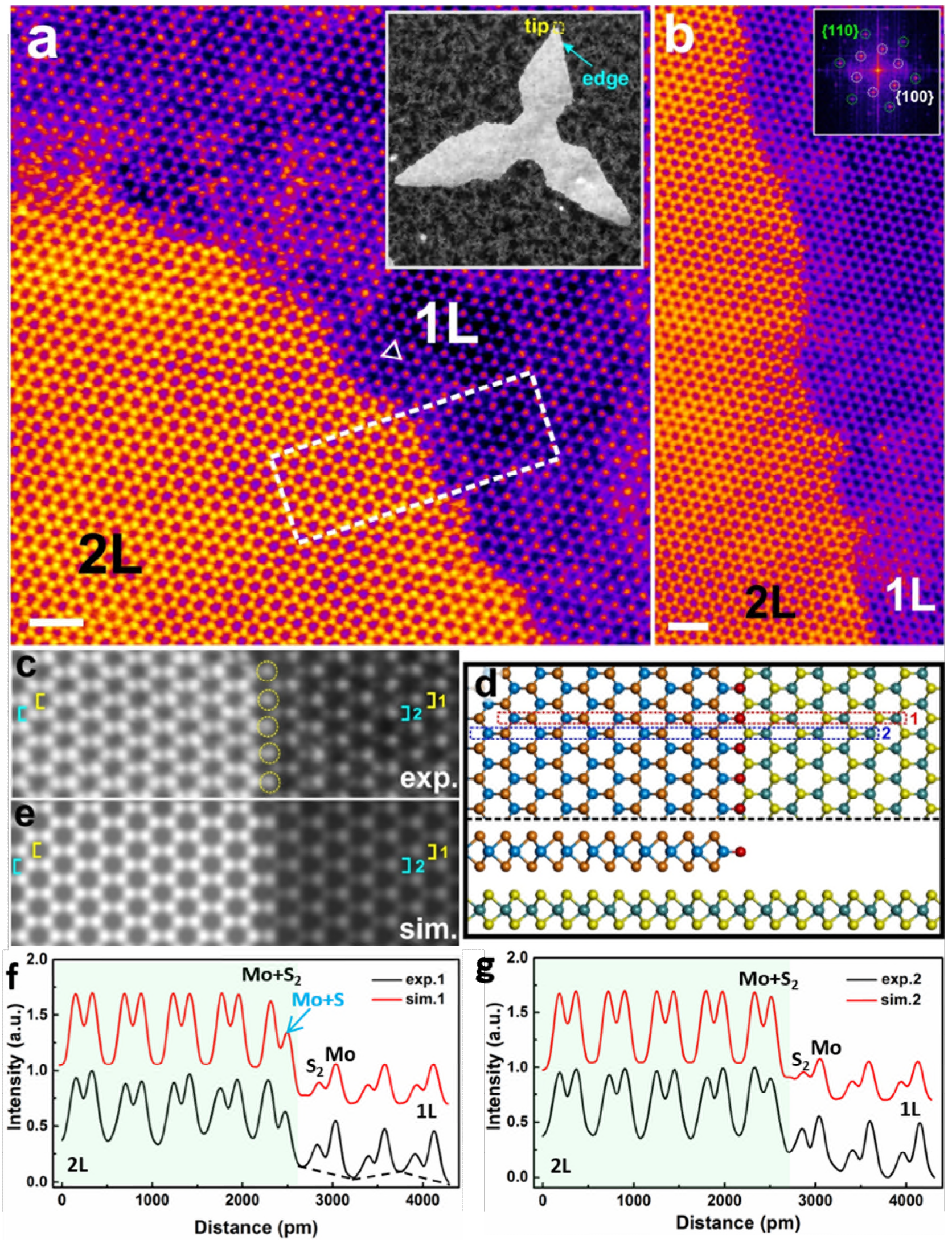

Figure 2. HAADF-STEM image showing the $2^{\text {nd }}$-layer step edge configuration of AA' stacked bilayer

$\mathbf{M o S}_{2}$. (a) HAADF-STEM image of the tip region of AA' stacked $2^{\text {nd }}$-layer $\mathrm{MoS}_{2}$, corresponding to the area 
marked by the yellow box in the inset. The lattice orientation of the $1^{\text {st }}$-layer $\operatorname{MoS}_{2}$ is indicated by the small white triangle. (b) HAADF-STEM image showing the extended $2^{\text {nd }}$-layer $\mathrm{MoS}_{2}$ step edge, corresponding to the region indicated by the cyan arrow in the inset to (a). Power spectrum calculated from (b) is shown inset. (c) Magnified image of the white-boxed region in (a). (d) Atomic model based on (c) showing both projection and side views. Single $\mathrm{S}$ atoms at the Mo-terminated zigzag edge of the $2^{\text {nd }}$-layer $\mathrm{MoS}_{2}$ are highlighted in red. (e) Multislice HAADF-STEM image simulation corresponding to the atomic model in (d) (see simulation parameters in Methods). (f) Intensity line profiles measured from the selected area labelled 1 (yellow square brackets) in (c) and (e), respectively. The structure corresponds to the region marked by the red dashed box in (d). (g) Intensity line profiles measured from the selected area labelled 2 (cyan square brackets) in (c) and (e), respectively. The structure corresponds to the region marked by the blue dashed box in (d). Green shading in both (f) and (g) indicates the region of $2^{\text {nd }}-$ layer $\mathrm{MoS}_{2}$. All scale bars correspond to $1 \mathrm{~nm}$.

Figure 3a shows the step edge of the other type of orientated three-pointed shuriken-shaped $2^{\text {nd }}$-layer $\mathrm{MoS}_{2}$, corresponding to domains outlined by the yellow dashed triangle in Figure $1 \mathrm{~b}$, which are rotated by $60^{\circ}$ with respect to the $2^{\text {nd }}$-layer $\mathrm{MoS}_{2}$ domain shown in the inset of Figure $2 \mathrm{a}$. The lattice direction for the $1^{\text {st }}$-layer $\mathrm{MoS}_{2}$ in Figure $3 \mathrm{a}$ is the same as that in Figure $2 \mathrm{a}$, as indicated by the small yellow and white triangles in the corresponding images. This confirms the single crystalline nature of the bottom $1^{\text {st }}$-layer $\mathrm{MoS}_{2}$. The step edge is parallel to the zigzag direction with small terraces. Figure $3 b$ shows the domain tip geometry, which has a truncated cusp consisting of three sides oriented by $120^{\circ}$. All three edges at the tip are aligned to three zigzag directions of the $\mathrm{MoS}_{2}$ lattice. Figure $3 \mathrm{c}$ shows a magnified HAADF-STEM image of the region defined by the orange box in Figure 3a with corresponding atomic models and multislice HAADF simulation shown in Figure $3 \mathrm{~d}$ and $3 \mathrm{e}$, respectively. Intensity line profiles along the armchair lattice direction across the $2^{\text {nd }}$-layer step edge in both experimental and simulated HAADF images are shown in Figure $3 \mathrm{f}$ and $3 \mathrm{~g}$, which shows three different atomic columns with different contrast. These are assigned as $2 \mathrm{~S}$, Mo and $2 \mathrm{~S}+\mathrm{Mo}$ from the lowest to the highest contrast, respectively. In addition, orientation of the two layers is staggered, with atoms observed in the hollow centers of hexagonal rings in projection consistent with $\mathrm{AB}$ stacking. 
The $2^{\text {nd }}$-layer step edge adopts the Mo-terminated zigzag configuration, which aligns with the same lattice direction as the $50 \% \mathrm{~S}$-covered Mo Klein edge observed in the AA' stacked $2^{\text {nd }}$-layer $\mathrm{MoS}_{2}$ but without dangling $\mathrm{S}$ atoms in the outermost row.

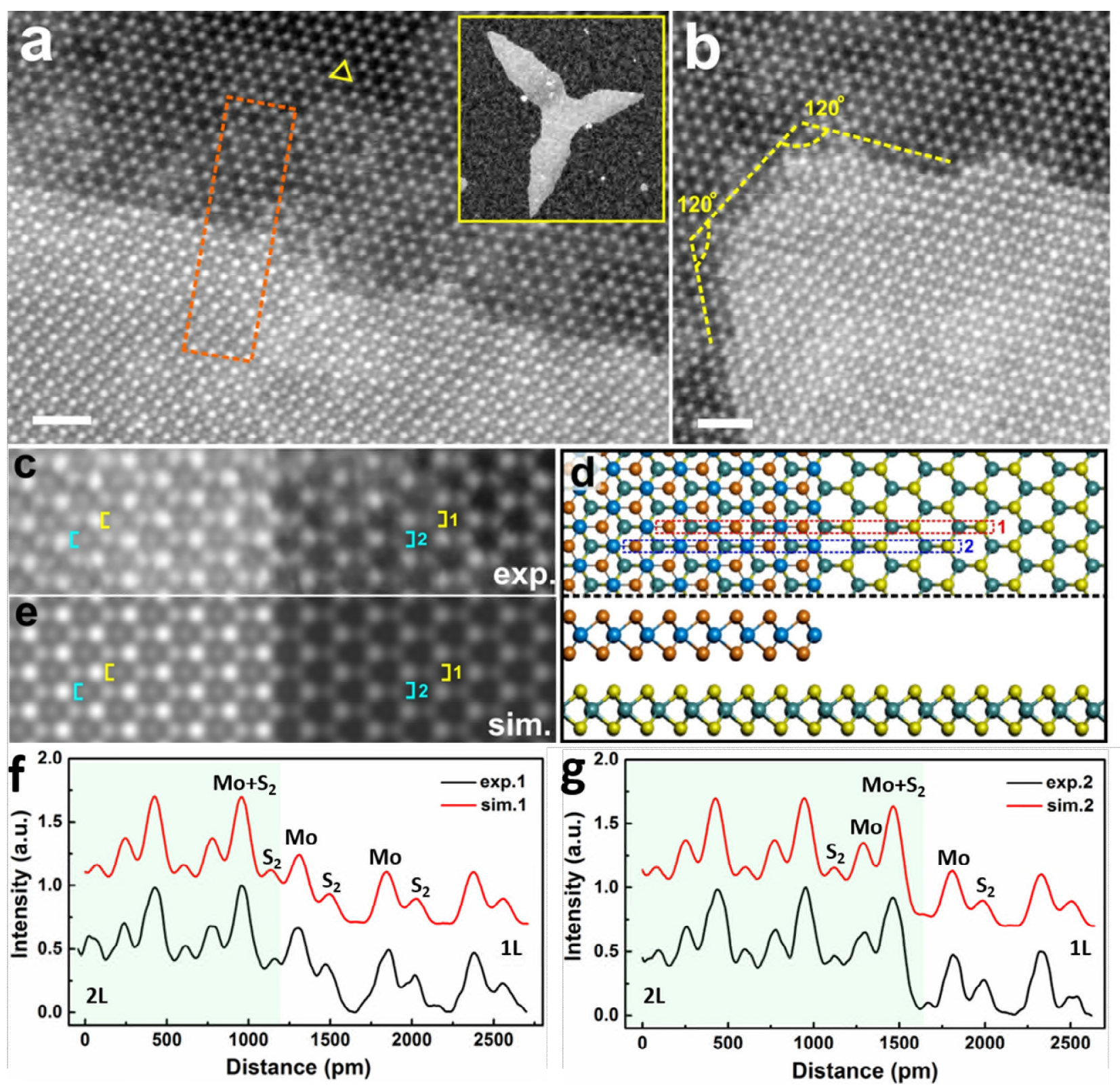

Figure 3. HAADF-STEM image showing the $2^{\text {nd }}$-layer step edge configuration of AB stacked bilayer MoS $_{2}$.

(a) HAADF-STEM image showing AB stacked $2^{\text {nd }}-$ layer $\mathrm{MoS}_{2}$. The lattice orientation of the $1^{\text {st }}$-layer $\mathrm{MoS}_{2}$ is marked by the yellow triangle in the same direction as the $1^{\text {st }}$-layer $\mathrm{MoS}_{2}$ in Figure 2a. Inset is a lowmagnification image showing the domain orientation of the $2^{\text {nd }}$-layer $\mathrm{MoS}_{2}$ in the $\mathrm{AB}$ stacking sequence, which 
is rotated by $60^{\circ}$ with respect to the $\mathrm{AA}^{\prime}$ stacked $2^{\text {nd }}$-layer domain shown inset to Figure 2a. (b) ADF-STEM image of the tip region of the $2^{\text {nd }}$-layer $\mathrm{MoS}_{2}$. (c) Magnified image of the orange boxed area in (a). (d) Atomic model based on (c) showing both projection and side views. (e) Multislice HAADF-STEM image simulation corresponding to the atomic model in (d) (see simulation parameters in Methods). (f) Intensity line profiles measured from the area labelled 1 (yellow squared brackets) in (c) and (e), respectively. The structure corresponds to the region marked by the red dashed box in $(\mathrm{d})$. (g) Intensity line profiles measured from the selected area labelled by 2 (cyan square brackets) in (c) and (e), respectively. The structure corresponds to the region marked by the blue dashed box in (d). Green shading in both (f) and ( $g$ ) indicates the region of $2^{\text {nd }}$-layer $\mathrm{MoS}_{2}$. All scale bars correspond to $1 \mathrm{~nm}$.

In Figure 4b, we show the relative occurrence of $\mathrm{AA}^{\prime}$ and $\mathrm{AB}$ stacking in bilayer $\mathrm{MoS}_{2}$, taking into account both the large shuriken-shaped domains and the small triangular nuclei. All of the shuriken-shaped domains and smaller triangular nuclei with defined size and shape obey either AA' or $\mathrm{AB}$ stacking sequences indicating an energetic advantage for these two stacking configurations under the growth conditions used. The ratio between $\mathrm{AA}^{\prime}$ and $\mathrm{AB}$ stacked domains is almost equal at $45 \%$ and 55\%, respectively, when considering both shuriken and triangular domains (Figure S2). It was observed that smaller nuclei are distributed in regions that are not close to larger shuriken-shaped domains, leaving a depletion area around each shuriken-shaped domain with a relatively low concentration of smaller nuclei (Figure 1b). It is also observed that, for closely situated pairs of shuriken-shaped domains with tips grown towards each other, the tips from two domains that grow head to head are shorter than the other tips in the same domain (Figure 4c-f). These two phenomena indicate that our shuriken-shaped $2^{\text {nd }}$-layer $\mathrm{MoS}_{2}$ were grown in conditions where the surface migration distance of active precursor species on the monolayer $\mathrm{MoS}_{2}$ substrate is the governing factor. This growth condition also can explain the formation of the shuriken geometry for $2^{\text {nd }}-1$ ayer $\mathrm{MoS}_{2}$. It is known that the surface curvature of crystal nuclei or liquid droplets could influence the speed of mass transfer, such as the crystal growth and the droplet evaporation, based on the Kelvin equation. ${ }^{47}$ For instance, in the crystal aging process, small nuclei with a convex surface and a higher surface 
curvature will spontaneously transfer their substances to larger crystals with a flatter surface and a lower surface curvature in the mother solution, resulting in the disappearance of small nuclei and the achievement of large crystals with more uniform sizes eventually. This can be explained by an increase of the surface energy with increasing degree of surface curvature. However, our case is opposite to the example above, as the tip with a larger convex surface curvature in a triangular $2^{\text {nd }}-$ layer $\mathrm{MoS}_{2}$ nucleus grows faster than the side, leading to formation of shuriken-shaped large crystals. This can be interpreted by a simple kinetic model illustrated in Figure 4g. As the growth is kinetically controlled by the transfer ability of precursor species, we can assume the longest migration distance of the precursor species absorbed on the $1^{\text {st }}$-layer $\mathrm{MoS}_{2}$ under this synthesis condition as ' $d$ ', meaning that only those precursor species locating within the distance of ' $d$ ' from the edges of a $2^{\text {nd }}$-layer $\mathrm{MoS}_{2}$ nucleus can move and attach to the nucleus. Then, we can select two points on the domain tip and side, respectively, and mark corresponding two regions where precursor feedstocks can effectively reach the $2^{\text {nd }}$-layer $\mathrm{MoS}_{2}$ edges, as indicated by the yellow and green areas on the left blue triangle in Figure 4g. It is clear that the point on the domain tip with a higher convex surface curvature obtains a larger sector region of precursor supplements than the point on the flat domain side, leading to a faster growth rate of the tip and finally resulting in the crystal geometry with extended tips for the $2^{\text {nd }}-$ layer $\operatorname{MoS}_{2}$. When two nuclei situated close together with two tips growing along the same axis in opposite directions, the regions of precursor supplements at two tips could overlap (yellow and red regions in Figure $4 \mathrm{~g}$ ), leading to a competition for the precursor species between them. This results in less precursor supply to these two tips compared to others, causing slower growth rate and eventually shorter tip lengths. The other geometric feature of note in the shuriken-shaped domains is the specific side curvature, as shown by the yellow dashed lines along the domain boundaries in Figure 4c and 4d. This boundary has several sharp deflections close to $120^{\circ}$ and differentiates the shuriken-shaped second layers from the previously observed three-pointed star-shaped monolayer $\mathrm{MoS}_{2}$ grown on amorphous $\mathrm{SiO}_{2} / \mathrm{Si}$ substrates, ${ }^{31,48}$. This shape could also be rationalised by the effect of growth on a 
crystalline $1^{\text {st }}$-layer $\mathrm{MoS}_{2}$. Since step edges along certain lattice directions (e.g. the Mo zigzag direction) might have a lower total energy, the $2^{\text {nd }}$-layer domains contain several sharp deflections with angles that sustain the preferable step edge orientations (e.g. $\left.60^{\circ}, 120^{\circ}\right)$.

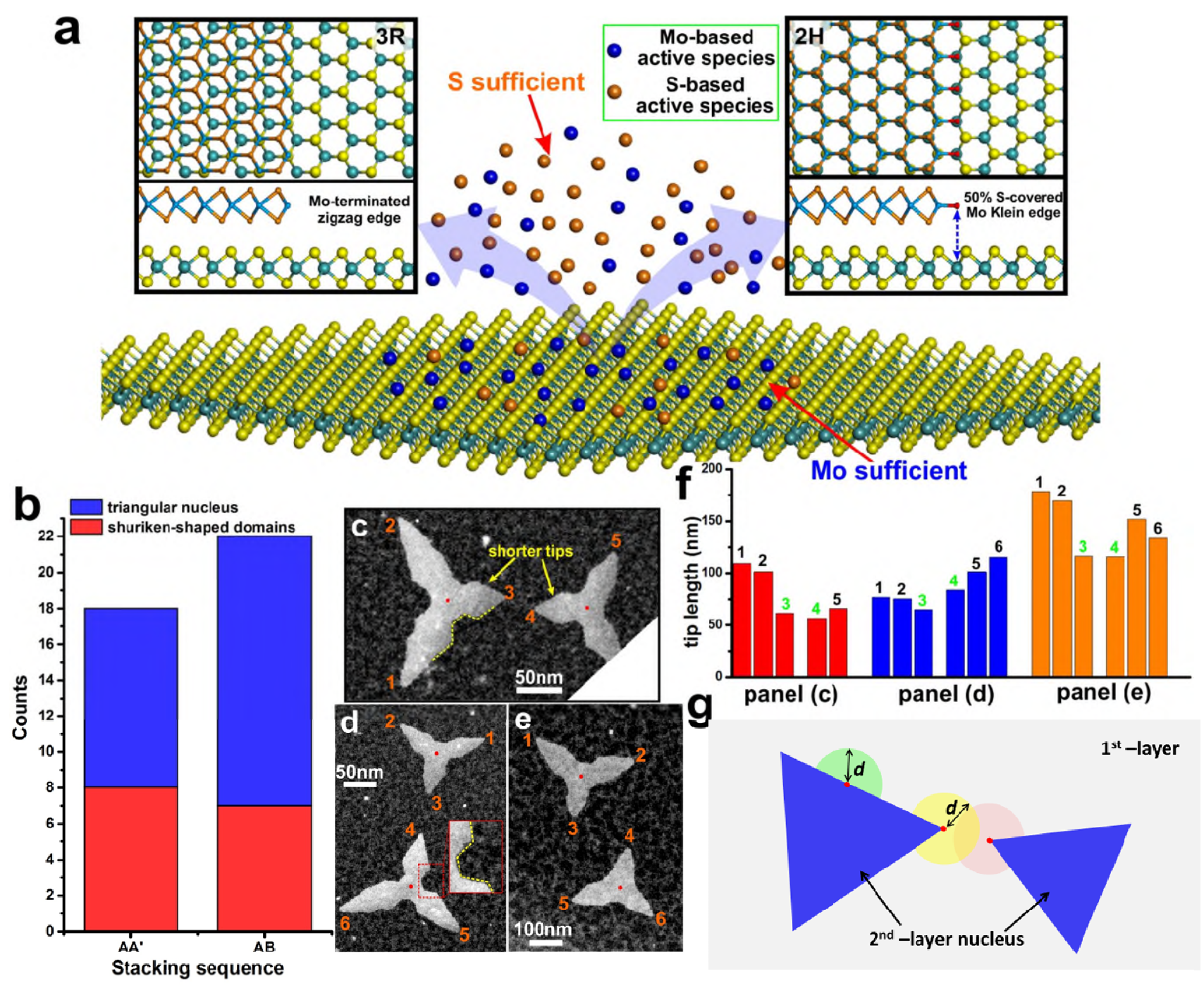

Figure 4. (a) Schematic illustration showing how a single layer $\mathrm{MoS}_{2}$ substrate and different stacking sequences impact $2^{\text {nd }}$-layer step edge configurations. (b) Statistical plot showing the relative distribution of $A A^{\prime}$ and $A B$ stacking sequences for bilayer $\mathrm{MoS}_{2}$, considering both large shuriken-shaped domains and small triangular nuclei. (c-e) Low magnification HAADF-STEM images showing the domain geometry of three different pairs of shuriken-shaped $\mathrm{MoS}_{2}$ second layers. The tips from two closely separated domains growing head to head are 
shorter than the other tips. Insets show magnified images of the red-boxed region highlighting a special boundary morphology with several $\sim 120^{\circ}$ deflections. (f) Histograms of tip length variations in different domains in panels (c) to (e), showing a general phenomenon that head-to-head grown domain tips are shorter than their counterparts in the same domain. Different columns correspond to domain tips marked with the same number in panels (c) to (e). Red, blue and orange column colors indicate tips from (c), (d) and (e), respectively. (g) Schematic illustration of a potential formation mechanism of shuriken-shaped $\mathrm{MoS}_{2} 2^{\text {nd }}$-layers on monolayer $\mathrm{MoS}_{2}$, with the grey background indicating the $1^{\text {st }}$-layer $\mathrm{MoS}_{2}$. The green sector represents the region of precursor supplements for a point on the side of the $2^{\text {nd }}$-layer nucleus, while the yellow and the red sectors represent the regions of precursor supplements for the point on the tip of two $2^{\text {nd }}$-layer nuclei, respectively. All sectors have a radius of ' $d$ '.

In summary, we have shown that the mixed AA' and $\mathrm{AB}$ stacking of bilayer seeds of $\mathrm{MoS}_{2}$ on larger $\mathrm{MoS}_{2}$ monolayer single crystals have $60^{\circ}$ rotation on the lattice orientation. Mixed AA' and AB stacked bilayers are expected due to the small energy difference and subsequent thermodynamic distribution. The presence of two orientated lattice directions within the secondary domain nuclei will have impact on any final bilayer crystal produced when these merge, where the $60^{\circ}$ rotation will lead to an anti-phase boundary (inversion boundary). This indicates that creating pure single crystal bilayer by the merger of seed nuclei on the surface of a pre-existing monolayer TMD may be challenging. These insights gained here were only possible by using HAADF-STEM to provide elemental discrimination of Mo and S sites and show the Mo zig-zag orientated edges of both triangular shaped domains.

\section{Methods}

Synthesis and transfer of monolayer $\mathrm{MoS}_{2}$ 
Epitaxially-grown three-pointed shuriken-shaped $\mathrm{MoS}_{2}$ bilayers were synthesized using a similar approach to that previously reported. ${ }^{49}$ Molybdenum trioxide $\left(\mathrm{MoO}_{3}, \geq 99.5 \%\right.$, Sigma-Aldrich) and sulphur ( $\mathrm{S}, \geq 99.5 \%$, Sigma-Aldrich) powder were used as precursors to grow bilayers on $\mathrm{SiO}_{2} / \mathrm{Si}$ substrates $\left(300 \mathrm{~nm}\right.$ thick $\left.\mathrm{SiO}_{2}\right)$ through a hydrogen-free chemical vapor deposition method (CVD) under atmospheric pressure. Two furnaces were used to give close independent control of temperature of the two precursors and the substrate. The heating temperature for $\mathrm{S}, \mathrm{MoO}_{3}$ and the $\mathrm{SiO}_{2} / \mathrm{Si}$ substrate were $\sim 180, \sim 300$, and $\sim 800^{\circ} \mathrm{C}$, respectively, with argon used as the carrier gas. To avoid the erosion of the $\mathrm{MoO}_{3}$ powder by the $\mathrm{S}$ vapor, the $\mathrm{MoO}_{3}$ powder was placed in an inner tube having a smaller diameter, which was then inserted into the larger 1-inch quartz tube. The S powder was loaded in the outer quartz tube. To increase the yield of bilayers, the growth time was increased by 5 minutes from the original recipe, thus increasing the nucleation density of second layers on monolayer MoS2. MoS2 was transferred onto the $\mathrm{Si}_{3} \mathrm{~N}_{4}$ TEM grid (Agar Scientific AG21580) through a standard polymerbased method. The surface of the $\mathrm{MoS}_{2} / \mathrm{SiO}_{2} / \mathrm{Si}$ substrate was first spin-coated with a thin membrane of poly (methyl methacrylate) (PMMA) and then floated on the surface of a $1 \mathrm{~mol} / \mathrm{L}$ potassium hydroxide $(\mathrm{KOH})$ solution to remove $\mathrm{SiO}_{2}$. After the $\mathrm{PMMA} / \mathrm{MoS}_{2}$ film was detached from the $\mathrm{Si}$ substrate, it was rinsed in deionized water several times and transferred to a holey $\mathrm{Si}_{3} \mathrm{~N}_{4}$ grid. It was then air-dried and subsequently baked at $180{ }^{\circ} \mathrm{C}$ for 15 minutes. The grid was eventually submerged in acetone for 8 hours to dissolve the PMMA.

\section{Scanning transmission electron microscopy and image processing}

Room temperature HAADF-STEM imaging was performed on an aberration corrected JEOL ARM300CF STEM equipped with a JEOL ETA corrector ${ }^{50}$ operated at an accelerating voltage of $80 \mathrm{kV}$ located in the electron Physical Sciences Imaging Centre (ePSIC) at Diamond Light Source. The convergence semi-angle used was $24.6 \mathrm{mrad}$ with a camera length of $20 \mathrm{~cm}$, which gave an annular recording range of $39-156 \mathrm{mrad}$ at the detector plane. The electron probe diameter was focused to 
$\sim 52 \mathrm{pm}$ with a dwell time ranging from $10-20 \mu$ s per pixel for imaging. The beam current was measured as $23 \mathrm{pA}$ using a Pico ampere meter.

Images were processed using the ImageJ software. They were processed by applying a Gaussian blur ( 1-2 pixels) for smoothing. SFor some grayscale HAADF-STEM images a fire false color LUT was applied to improve the visualisation. Atomic models were generated using the Accelrys Discovery Studio Visualizer. Multislice HAADF image simulation based on the corresponding atomic models were carried out using JEMS software with parameters corresponding to the imaging condition. For Figure 2e, Figure S1c and Figure S1f, key simulation parameters, including defocus spread, defocus and spherical aberration (Cs), were set to be $5.3 \mathrm{~nm}, 16 \mathrm{~nm}$ and $0.1 \mathrm{~mm}$, respectively, while for Figure $3 \mathrm{e}$, these three parameters were $5.3 \mathrm{~nm}, 14 \mathrm{~nm}$ and $0.08 \mathrm{~mm}$, respectively.

Conflict of Interest: The authors declare no competing financial interest.

\section{Acknowledgements}

JHW thanks the Royal Society for support. SW thanks the China Scholarship Council for support. AIK acknowledges EPSRC support under platform grant EP/K032518/1.

\section{References}

1 B. Radisavljevic, A. Radenovic, J. Brivio, V. Giacometti and A. Kis, Nat. Nanotechnol., 2011, 6, 147-150.

2 Q. H. Wang, K. Kalantar-Zadeh, A. Kis, J. N. Coleman and M. S. Strano, Nat. Nanotechnol., 2012, 7, 699-712.

3 R. Ganatra and Q. Zhang, ACS Nano, 2014, 8, 4074-4099.

4 M. Amani, M. L. Chin, a. G. Birdwell, T. P. O’Regan, S. Najmaei, Z. Liu, P. M. Ajayan, J. Lou and M. Dubey, Appl. Phys. Letts., 2013, 102, 193107.

$5 \quad$ J. He, K. Hummer and C. Franchini, Phys. Rev. B, 2014, 89, 075409. 

Letts., 2010, 10, 1271-5.

H. Li, Q. Zhang, C. C. R. Yap, B. K. Tay, T. H. T. Edwin, A. Olivier and D. Baillargeat, Adv. Funct. Mater., 2012, 22, 1385-1390.

8 Y. Yu, S. Y. Huang, Y. Li, S. N. Steinmann, W. Yang and L. Cao, Nano Letts., 2014, 14, $553-558$.

9 W. Bao, X. Cai, D. Kim, K. Sridhara and M. S. Fuhrer, Appl. Phys. Letts., 2013, 102, 042104.

10 G.-H. Lee, Y.-J. Yu, X. Cui, N. Petrone, C.-H. Lee, M. S. Choi, D.-Y. Lee, C. Lee, W. J. Yoo, K. Watanabe, T. Taniguchi, C. Nuckolls, P. Kim and J. Hone, ACS Nano, 2013, 7, 7931-7936.

11 D. J. Late, Y. K. Huang, B. Liu, J. Acharya, S. N. Shirodkar, J. Luo, A. Yan, D. Charles, U. V. Waghmare, V. P. Dravid and C. N. R. Rao, ACS Nano, 2013, 7, 4879-4891. Zhang, Small, 2012, 8, 63-7.

13 A. Ramasubramaniam, D. Naveh and E. Towe, Phys. Rev. B, 2011, 84, 205325.

14 Q. Liu, L. Li, Y. Li, Z. Gao, Z. Chen and J. Lu, J. Phys. Chem. C, 2012, 116, 21556-21562.

15 T. Chu, H. Ilatikhameneh, G. Klimeck, R. Rahman and Z. Chen, Nano Letts., 2015, 15, $8000-8007$.

R. Suzuki, M. Sakano, Y. J. Zhang, R. Akashi, D. Morikawa, A. Harasawa, K. Yaji, K. Kuroda, K. Miyamoto, T. Okuda, K. Ishizaka, R. Arita and Y. Iwasa, Nat. Nanotechnol., $2014,9,611-617$. 
J. Yan, J. Xia, X. Wang, L. Liu, J. L. Kuo, B. K. Tay, S. Chen, W. Zhou, Z. Liu and Z. X. Shen, Nano Letts., 2015, 15, 8155-8161.

K. Liu, L. Zhang, T. Cao, C. Jin, D. Qiu, Q. Zhou, A. Zettl, P. Yang, S. G. Louie and F. Wang, Nat. Commun., 2014, 5, 4966.

A. Yacoby, Nat. Phys., 2011, 7, 925-926.

21 I. Song, C. Park and H. C. Choi, RSC Adv., 2015, 5, 7495-7514.

A. M. van der Zande, J. Kunstmann, A. Chernikov, D. a Chenet, Y. You, X. Zhang, P. Y. Huang, T. C. Berkelbach, L. Wang, F. Zhang, M. S. Hybertsen, D. a Muller, D. R. Reichman, T. F. Heinz and J. C. Hone, Nano Letts., 2014.

23 P. C. Yeh, W. Jin, N. Zaki, J. Kunstmann, D. Chenet, G. Arefe, J. T. Sadowski, J. I. Dadap, P. Sutter, J. Hone and R. M. Osgood, Nano Letts., 2016, 16, 953-959.

24 K. Kang, S. Xie, L. Huang, Y. Han, P. Y. Huang, K. F. Mak, C.-J. Kim, D. Muller and J. Park, Nature, 2015, 520, 656-660.

25 S. Wang, M. Pacios, H. Bhaskaran and J. H. Warner, Nanotechnology, 2016, 27, 8560485611.

J. Zhang, H. Yu, W. Chen, X. Tian, D. Liu, M. Cheng, G. Xie, W. Yang, R. Yang, X. Bai, D. Shi and G. Zhang, ACS Nano, 2014, 8, 6024-6030.

27 V. Senthilkumar, L. Tam, Y. Kim and Y. Sim, Nano Res., 2014, 7, 1759-1768.

28 Y. W. Son, M. L. Cohen and S. G. Louie, Phys. Rev. Letts., 2006, 97, 216803.

29 Y.-W. Son, M. L. Cohen and S. G. Louie, Nature, 2006, 444, 347-349.

30 Z. Luo, S. Kim, N. Kawamoto, A. M. Rappe and a T. C. Johnson, ACS Nano, 2011, 5, 915460. 
31 S. Wang, Y. Rong, Y. Fan, M. Pacios, H. Bhaskaran, K. He and J. H. Warner, Chem. Mater., 2014, 26, 6371-6379.

E. Erdogan, I. H. Popov, A. N. Enyashin and G. Seifert, Eur. Phys. J. B, 2012, 85, 33-36.

Chorkendorff and J. K. Nørskov, J. Am. Chem. Soc., 2005, 127, 5308-5309.

35 Y. Li, Z. Zhou, S. Zhang and Z. Chen, J. Am. Chem. Soc., 2008, 130, 16739-44.

36 K. He, G.-D. Lee, A. W. Robertson, E. Yoon and J. H. Warner, Nat. Commun., 2014, 5, 3040.

37 K. He, A. W. Robertson, S. Lee, E. Yoon, G. Lee and J. H. Warner, ACS Nano, 2014, 8, $12272-12279$.

38 K. Kim, S. Coh, C. Kisielowski, M. F. Crommie, S. G. Louie, M. L. Cohen and A. Zettl, Nat. Commun., 2013, 4, 2723.

39 W. Zhou, X. Zou, S. Najmaei, Z. Liu, Y. Shi, J. Kong, J. Lou, P. M. Ajayan, B. I. Yakobson and J.-C. Idrobo, Nano Letts., 2013, 13, 2615-2622.

41 O. L. Krivanek, M. F. Chisholm, V. Nicolosi, T. J. Pennycook, G. J. Corbin, N. Dellby, M. F. Murfitt, C. S. Own, Z. S. Szilagyi, M. P. Oxley, S. T. Pantelides and S. J. Pennycook, Nature, $2010,464,571-574$. Warner, ACS Nano, 2016, 10, 9831-9839. 
44 A. M. van der Zande, P. Y. Huang, D. A. Chenet, T. C. Berkelbach, Y. You, G.-H. Lee, T. F. Heinz, D. R. Reichman, D. A. Muller and J. C. Hone, Nat. Mater., 2013, 12, 554-561.

45 H. Schweiger, P. Raybaud, G. Kresse and H. Toulhoat, J. Catal., 2002, 207, 76-87.

46 A. Govind Rajan, J. H. Warner, D. Blankschtein and M. S. Strano, ACS Nano, 2016, 10, $4330-4344$.

47 D. J. Woodland and E. Mack Jr., J. Am. Chem. Soc., 1933, 55, 3149-3161.

48 Y.-H. Lee, X.-Q. Zhang, W. Zhang, M.-T. Chang, C.-T. Lin, K.-D. Chang, Y.-C. Yu, J. T.W. Wang, C.-S. Chang, L.-J. Li and T.-W. Lin, Adv. Mater., 2012, 24, 2320-5.

49 S. Wang, X. Wang and J. H. Warner, ACS Nano, 2015, 9, 5246-5254.

50 F. Hosokawa, H. Sawada, Y. Kondo, K. Takayanagi and K. Suenaga, Microscopy, 2013, 62, $23-41$

TOC graphic

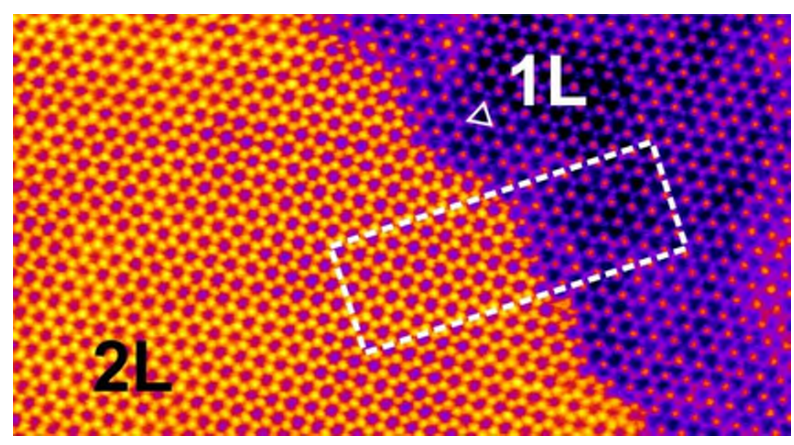

